\title{
Eficacia de las Asesorías Grupales en el Rendimiento Académico de los estudiantes que asisten al Comité Central de Tutorías y Asesorías de la FMH - USMP
}

Mauro Rivera Ramírez', Luis Roble Áleman ${ }^{2}$, María Elizabeth Flores Vásquez ${ }^{3}$, María Teresa Rivera Encinas ${ }^{4}$, Karina D. García De la Puente ${ }^{5}$

\section{RESUMEN}

Objetivo: Determinar la eficacia de las asesorías grupales en el rendimiento académico de los alumnos participantes en el Semestre 2013 - I.

Material y Métodos: Se compararon las notas obtenidas antes y después de recibir Asesorías Grupales. La muestra incluyó a los estudiantes que asistieron en forma voluntaria. Participaron 223 estudiantes en las asesorías de Matemática aplicada a la Medicina, Bioestadística, Química Médica y Filosofía.Se utilizó el paquete estadístico SPSS versión 22. Se determinó la normalidad de los datos mediante la Prueba de Kolmogorov-Sminov, utilizando T de Student para muestras relacionadas, en el caso de la data paramétrica; y la Prueba de Rangos de Wilcoxon, para las no paramétricas.

Resultados: En el curso de Filosofía, el promedio de la nota de los participantes del Primer Parcial fue de 9.58, mientras que la del Segundo Parcial fue de 12.89, existiendo una mejora de 3.3 puntos. Esta diferencia se analizó con la Prueba de Rangos de Wilcoxon, encontrando significancia. En el curso de Química Médica, la media de la nota del Primer Parcial fue 12.5; 0.88 puntos menores a la media del Segundo Parcial (13.39). Con significancia estadística por la Prueba T de Student para muestras relacionadas. Existió evidencia para afirmar que en el curso de Matemática aplicada a la Medicina, los 4.89 puntos a favor son estadísticamente válidos. En Bioestadística se halló un incremento de sólo 0.88 puntos en la comparación de medias, no pudiéndose demostrar evidencia estadística.

Conclusiones: Las estrategias del Programa de Asesorías organizado por la Central de Tutorías durante el Semestre 2013-I tuvo impacto positivo en el rendimiento académico de los alumnos que voluntariamente asistieron.

(Horiz Med 2014; 14(1): 11-17)

Palabras clave: asesoría, proceso educativo, rendimiento (Fuente: DeCS BIREME)

Effectiveness of group counseling on the academic performance of students attending the Central Committee of tutorials of the FMH-USMP

\footnotetext{
ABSTRACT for nonparametric. comparison of averages were found, so it could not demonstrate statistical evidence.

Key words: advice, educational process, performance (Source: MeSH NLM)

Presidente del Comité Central de Tutorías y Asesorías. Médico - cirujano.

Miembro del Comité Central de Tutorías y Asesorías. Médico - cirujano.

Miembro del Comité Central de Tutorías y Asesorías. Licenciada en Psicología.

Miembro del Comité Central de Tutorías y Asesorías. Médico - cirujano.

5 Miembro del Comité Central de Tutorías y Asesorías. Médico - cirujano.
}

Objective: To determine the effectiveness of Academic Advising Group on academic performances in the semester 2013 - I. Material and Methods: We compared the grades that were obtained before and after receiving academic group advice. The sample included students who voluntarily attended to the classes. 223 students participated in the Mathematics applied to MedicineClass, Biostatistics, Medical Chemistry and Philosophy. SPSS version 22 was used. Normality of data was determined by the Kolmogorov - Sminov Test, using $T$-test for related samples in the case of parametric data, and Wilcoxon Rank test

Results: In Philosophy, the average rate on the First Exam was 9.58. In contrast, on the Second Exam it was $12.89,3.3$ points more. This difference was analyzed with the Wilcoxon Rank Test, finding significance. In Medical Chemistry, the average on the First Exam was 12.5, 0.88 points lower than the average of the Second Part, which was 13.39. We found that this difference is statistically significant using the $T$ test for paired samples. In addition, it showed that there is evidence to say that in Mathematics, the 4.89 points higher were statistically valid. In Biostatistics an increase of only 0.88 points in the

Conclusions: Strategies, that were organized by the Advising Tutoring Center during Semester 2013 -I, had positive impact on the academic performance of students who voluntarily attended. (Horiz Med 2014; 14(1): 11-18) 


\section{INTRODUCCIÓN}

El Comité Central de Tutorías y Asesorías es un grupo multidisciplinario de personas que trabajan como facilitadores del aprendizaje $(1,2)$, que tienen como funciones intervenir de forma articulada, en cuanto a la enseñanza y trasmisión de contenidos científicos, facilitar la adquisición de competencias en conocimientos, procedimientos y actitudes, y principalmente, tener como eje principal al Estudiante de Medicina (1). Es por ello, que los tutores, al descubrir problemas bio-psico-socio-académicos que afecten el rendimiento de los alumnos durante la carrera de Medicina Humana, se le da a conocer estrategias concretas, para que desarrollen autonomía en el manejo de sus estudios y de su conducta para el logro de objetivos y la excelencia profesional. (3-6).

El Docente, como Tutor-Asesor, cumple un papel trascendental en la mejora del rendimiento académico de los alumnos de la Facultad de Medicina Humana de la Universidad de San Martín de Porres (4), teniendo como finalidad esencial la formación integral del estudiante, a través de una serie de instrumentos y actividades semestrales, como, encuestas, clases de reforzamiento y tutorías (1). Con ello, se logra mej orar el rendimiento académico de los alumnos que acuden periódicamente, haciendo hincapié en aquellos que presentan deficiencias académicas.

Cuando los al umnos perciben que tienen las habilidades y estrategias de aprendizaje para tener éxito, es más probable que deseen continuar y terminar sus estudios (Hendriksen, Yang, Love y Hall, 2005). El tutor debe favorecer el aprendizaje de los alumnos, ser guía, orientador y proveer las herramientas necesarias para que generen su propio conocimiento. Al respecto, Cruz, Echeverría y Vales (2008) comentan que una de las actividades prioritarias de la acción tutorial es el desarrollo de estrategias, como la organización del texto, programación habilidades de estudio e incrementar su desempeño académico.

Bajo esta perspectiva, el Comité Central de Tutorías y Asesorías, ayuda a que los estudiantes logren un aprovechamiento óptimo en su desempeño académico a lo largo de todo el proceso formativo; siendo la Orientación Tutorial la ayuda que a muchos les falta, para el proceso de maduración vocacional del estudiante conduciéndolo a tomar decisiones y gestionar su proyecto profesional (1).

Los integrantes del Comité Central de Tutorías y Asesorías realizan constantemente difusión de lo que se hace en beneficio del alumno.

Para las funciones propias de Asesorías, se utilizan las aulas asignadas por el Departamento Académico de Ciencias Básicas y los ambientes propios del Comité, participan los docentes de las diferentes asignaturas según las necesidades de los estudiantes con 0 sin deficiencia académica, ya que algunos alumnos, solo quieren reforzar sus conocimientos a pesar de no tener problemas en sus estudios.

Este trabajo de investigación resulta ser necesario para conocer la efectividad del trabajo de asesorías grupales del Comité Central de Tutorías y Asesorías en el rendimiento académico y, con ello, demostrar el beneficio para los estudiantes.

\section{MATERIAL Y MÉTODOS}

Se incluyeron en el estudio, a todos los alumnos matriculados en el Primer y Segundo año de la carrera de Medicina Humana de la USMP en el ciclo 2013-I, que asistieron voluntariamente a 2 asesorías grupales organizadas por la Central de Tutorías y Asesorías.

Participaron 223 estudiantes en total, 144 mujeres y 79 varones; entre 16 y 36 años de edad. Del total de participantes, 64 asistieron a las tutorías en Matemática aplicada a la medicina, 30 a Bioestadística, 129 a Química médica y 18 a Filosofía.

\section{Intervención}

Se realizaron 2 asesorías grupales teórico-prácticas por cada Curso (Filosofía, Química Médica, Matemática Aplicada a la medicina y Bioestadística), en las semanas previas al Segundo Examen Parcial. Cada asesoría grupal, tuvo una duración de 2 horas y fue dirigida por un Docente perteneciente a cada cátedra mencionada. 


\section{Metodología}

En la Facultad de Medicina de la USMP, se califica en base a 2 exámenes teóricos cancelatorios, con el mismo nivel de dificultad (2).

Para el primer examen, no se realizaron asesorías de reforzamiento, a diferencia, de lo sucedido para el segundo. Luego de finalizar el semestre académico 2013 - I, se solicitaron las notas del primer y segundo examen a la Oficina de Registros Académicos y se utilizó el programa Microsoft Excel 2010 para la organización de los datos y comparar los resultados obtenidos antes y después de las asesorías grupales organizadas por la Central de Tutorías y Asesorías.

Para el análisis de la información obtenida se utilizó la Herramienta estadística SPSS versión 22.

Se determinó la normalidad de los datos mediante la Prueba de Kolmogorov-Sminov, utilizando $T$ de student para muestras relacionadas en el caso de la data paramétrica y la Prueba de Rangos de Wilcoxon para las no paramétricas.

\section{RESULTADOS}

\section{Filosofía}

En las Asesorías grupales para el curso de Filosofía, participaron 18 alumnos de Primer año de Medicina Humana.

Después del análisis de los datos, se encontró que el promedio de la Nota de los participantes del Primer Parcial fue de 9.58, mientras que la del Segundo Parcial fue de 12.89 , existiendo una mejora de 3.3 puntos después de realizadas las Asesorías Grupales.

Se analizaron los datos mediante la Prueba de Kolmogorov-Sminov, determinando que la data no tiene una distribución normal (Tabla 1), motivo por el cual se utilizó la Prueba no paramétrica de Rangos de Wilcoxon (Tabla 2). Diferencia que existe entre la Primera y Segunda calificación es estadísticamente significativa $(p<0.05)$. Tabla 3
Tabla 1. Prueba de Kolmogorov-Sminov

\begin{tabular}{|c|c|c|c|c|}
\hline & & $\begin{array}{l}\text { NOTA DEL } \\
\text { PRIMER } \\
\text { EXÁMEN }\end{array}$ & $\begin{array}{l}\text { NOTA DEL } \\
\text { SEGUNDO } \\
\text { EXÁMEN }\end{array}$ & DIFERENCIA \\
\hline $\mathbf{N}$ & & 18 & 18 & 18 \\
\hline \multirow{2}{*}{$\begin{array}{l}\text { Parámetros } \\
\text { normales }\end{array}$} & Media & 9.583 & 12.889 & 3.3056 \\
\hline & $\begin{array}{l}\text { Desviación } \\
\text { estándar }\end{array}$ & 38050 & 2.2852 & 3.07810 \\
\hline \multirow{3}{*}{$\begin{array}{l}\text { Máximas } \\
\text { diferencias } \\
\text { extremas }\end{array}$} & Absoluta & -188 & -187 & -256 \\
\hline & Positivo & -188 & -185 & -135 \\
\hline & Negativo & -114 & -187 & -256 \\
\hline \multicolumn{2}{|c|}{ Estadístico de prueba } & -188 & -187 & -256 \\
\hline \multicolumn{2}{|c|}{ Sig. asintótica (bilateral) } & ,092 & ,098 &, 003 \\
\hline
\end{tabular}

Tabla 2: Rangos de WILCOXON: FILOSOFIA

\begin{tabular}{|c|c|c|c|c|}
\hline & & $\mathbf{N}$ & $\begin{array}{c}\text { Rango } \\
\text { promedio }\end{array}$ & $\begin{array}{c}\text { Suma de } \\
\text { rangos }\end{array}$ \\
\hline $\begin{array}{c}\text { NOTA DEL } \\
\text { SEGUNDO } \\
\text { EXAMEN }\end{array}$ & $\begin{array}{c}\text { Rangos } \\
\text { negativos }\end{array}$ & 3 & 3.83 & 11.50 \\
\hline \multirow{3}{*}{$\begin{array}{c}\text { NOTA DEL } \\
\text { PRIMER } \\
\text { EXAMEN }\end{array}$} & $\begin{array}{c}\text { Rangos } \\
\text { positivos }\end{array}$ & 15 & 10.63 & 159.50 \\
\hline & Empates & 0 & & \\
\hline & Total & 18 & & \\
\hline
\end{tabular}

Tabla 3. Estadísticos de prueba

NOTA DEL SEGUNDO

EXAMEN- NOTA DEL

PRIMER EXAMEN

Z

$-3,231$

Sig.

asintótica

(bilateral)
.001 


\section{QUÍMICA MÉDICA}

Participaron voluntariamente 129 alumnos del Primer año de Medicina Humana.

Tabla 4. Prueba de Kolmogorov-Sminov para una muestra: QUIMICA

\begin{tabular}{|c|c|c|c|c|}
\hline & & $\begin{array}{c}\text { NOTA DEL } \\
\text { PRIMER } \\
\text { EXÁMEN }\end{array}$ & $\begin{array}{c}\text { NOTA DEL } \\
\text { SEGUNDO } \\
\text { EXÁMEN }\end{array}$ & DIFERENCIA \\
\hline \multicolumn{2}{|l|}{$\mathbf{N}$} & 129 & 129 & 129 \\
\hline \multirow{2}{*}{$\begin{array}{l}\text { Parámetros } \\
\text { normales }\end{array}$} & Media & 12.508 & 13.391 & 0.8837 \\
\hline & $\begin{array}{l}\text { Desviación } \\
\text { estándar }\end{array}$ & 2.7078 & 3.5377 & 3.01008 \\
\hline \multirow{3}{*}{$\begin{array}{l}\text { Máximas } \\
\text { diferencias } \\
\text { extremas }\end{array}$} & Absoluta & .072 & .056 & .053 \\
\hline & Positivo & .053 & .049 & .053 \\
\hline & Negativo & -.072 & -.056 & -.052 \\
\hline \multicolumn{2}{|c|}{ Estadístico de prueba } & .072 & .056 & .053 \\
\hline \multicolumn{2}{|c|}{ Sig. asintótica (bilateral) } & 100 & 200 & 200 \\
\hline
\end{tabular}

Se encontró que la media de la Nota del primer Parcial fue 12.5. Es decir, 0.88 puntos menores a la media del Segundo Parcial, que fue de 13.39. Tabla 4.

Al aplicar la Prueba de normalidad, se llegó a la conclusión que la data tiene una distribución normal, por este motivo para comparar las medias, se utilizó una prueba paramétrica, Ilamada Prueba $\mathrm{T}$ de Student para muestras relacionadas.

Al analizar los datos, encontramos un valor $p$ de $0.001(p<0.05)$, lo que indica que la diferencia entre las medias de las notas antes y después de las Asesorías, es estadísticamente significativa. Tabla 5.

Tabla 5. Prueba de muestras emparejadas: QUIMICA

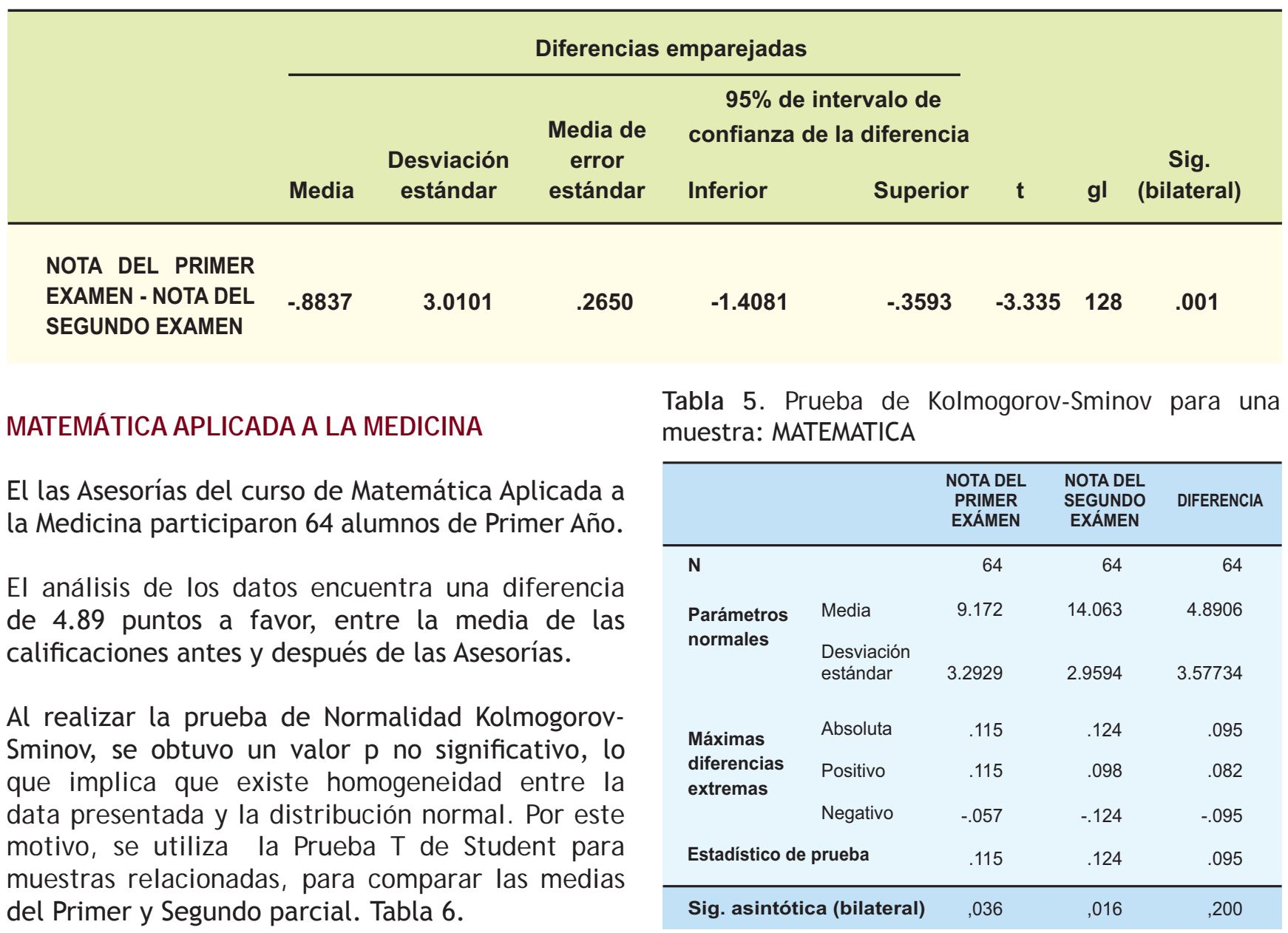


Al realizar la comparación de las medias se encontró un valor $p$ de $3.38649612608311 \mathrm{E}-16(\mathrm{p}<0.05)$, por lo que se rechaza la Hipótesis Nula de Igualdad y se concluye que la diferencia entre el promedio de la Primera y Segunda Calificación es estadísticamente significativa. Tabla 7.

\section{BIOESTADISTICA}

Asistieron a las Asesorías 30 estudiantes de Segundo año, matriculados en el ciclo 2003-I.

Se calcularon las medias de las calificaciones de los
Exámenes Parciales, antes y después de las Asesorías Grupales, encontrándose un valor de 14.083 para el primer parcial y 14.093 para el Segundo parcial, lo que implica un incremento de la media de 0.0097 puntos. Tabla 8.

Se realizó la Prueba de Kolmogorov-Sminov confirmando la normalidad de la data, por lo que se utilizó la Prueba $\mathrm{T}$ de student para muestras relacionadas, en la que se encontró un valor $p$ de 0.98710 , que implica que no existe evidencia de que la diferencia sea estadísticamente significativa. Tabla 9.

Tabla 7. Prueba de muestras emparejadas: MATEMATICA

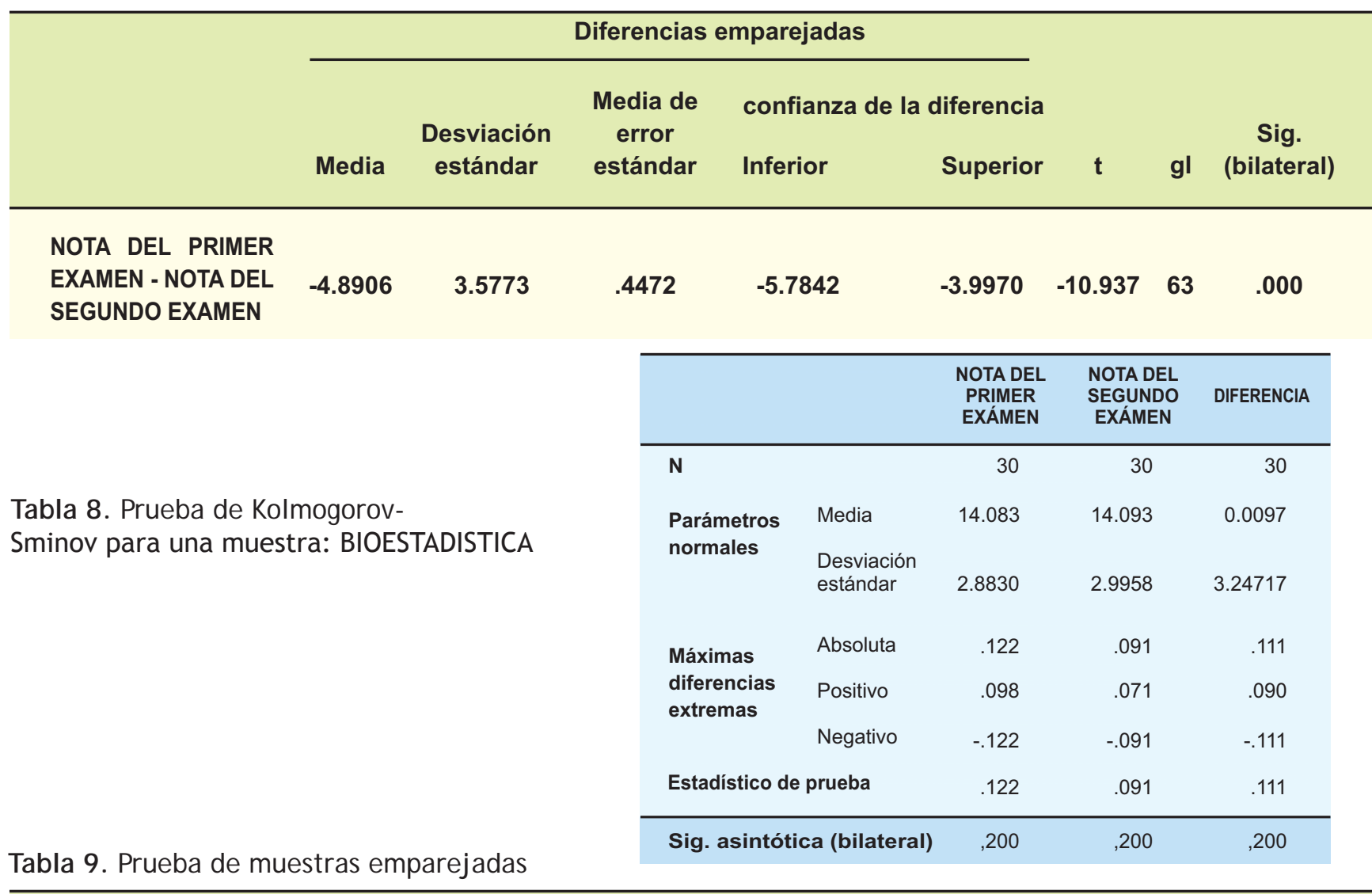

\begin{tabular}{|c|c|c|c|c|c|c|c|}
\hline & \multicolumn{4}{|c|}{ Diferencias emparejadas } & & & \\
\hline Media & $\begin{array}{c}\text { Desviación } \\
\text { estándar }\end{array}$ & $\begin{array}{c}\text { Media de } \\
\text { error } \\
\text { estándar }\end{array}$ & $\begin{array}{l}\text { confianz } \\
\text { Inferior }\end{array}$ & $\begin{array}{l}\text { diferencia } \\
\text { Superior }\end{array}$ & $t$ & gl & $\begin{array}{c}\text { Sig. } \\
\text { (bilateral) }\end{array}$ \\
\hline
\end{tabular}




\section{DISCUSIÓN}

Las tutorías y asesorías son modalidades de la actividad docente que comprenden un conjunto sistematizado de acciones educativas centradas en el estudiante (3), cuyos principales objetivos son: aumentar el rendimiento académico y disminuir la tasa de deserción de la Universidad.

Las tutorías tienen diferentes formas de intervención (3), en las que se utilizan espacios y tiempos adicionales para reforzar lo aprendido y resolver dudas específicas.

De esta forma, durante el ciclo 2013-I, se armaron programas educativos de reforzamiento con voluntarios, en cursos seleccionados de Primer y Segundo año.

Como se presenta en nuestro estudio, las estrategias del Programa de Asesorías, organizado por la Central de Tutorías durante el Ciclo 2013-I, tuvieron un impacto positivo en el rendimiento académico de los alumnos que voluntariamente asistieron a las 2 clases teórico-prácticas, siendo estadísticamente significativas en el caso de Filosofía, Química médica y Matemática aplicada a la Medicina.

En el caso de Bioestadística, el incremento en las calificaciones que se logró fue mínimo, probablemente debido a la mayor carga y dificultad académica que tiene un alumno de Segundo año en el Primer Semestre, en comparación con el de Primer año.

Cabe resaltar que, a pesar que hemos tratado que no existan sesgos, existieron variables intervinientes que no fueron posibles de controlar, como, el compromiso de los alumnos en el estudio de las materias, la diferencia que existió entre los alumnos repitentes que fueron a las asesorías y los alumnos nuevos, con respecto a la cantidad de cursos matriculados, el tiempo de estudio y el conocimiento previo de la materia. Sin embargo, los resultados de este estudio nos abrirán las puertas para el mejor monitoreo de las actividades del Comité Central de Tutorías y Asesorías, observando las amenazas y debilidades para transformarlas en fortalezas y oportunidades.
En conclusión, la presente investigación, nos ha permitido determinar la eficacia de las asesorías grupales en el contexto del rendimiento académico, así como, identificar cuáles son los programas que están funcionando de acuerdo a nuestros objetivos y cuáles requieren una reestructuración y actualización, ya que se conoce que existen otras herramientas a utilizar, como es el caso de la tutoría virtual $(7,9,10)$, que ha resultado un apoyo fundamental para las actividades de aprendizaje en general, que ya será tema de discusión para posteriores estudios.

\section{AGRADECIMIENTOS}

Al Dr. Frank Lizaraso Caparó, Decano de la Facultad de Medicina Humana de la USMP, por su invaluable ayuda y colaboración permanente. Un especial agradecimiento a todos los integrantes de la Oficina de Registros Académicos, puesto que sin su gran apoyo y paciencia, no hubiera sido posible este trabajo. Y, por supuesto, a todos los docentes asesores y alumnos, que gracias a su esfuerzo y dedicación nos inspiran a trabajar con ahínco para demostrar que sí se pueden alcanzar las metas trazadas.

\section{Fuentes de financiamiento}

Este trabajo fue auto financiado por los autores.

\section{Conflictos de interés}

Los autores declaran no tener conflictos de interés. 


\section{REFERENCIAS BIBLIOGRÁFICAS}

1. Reglamento del Comité Central de Tutorías y Asesorías de la FMH - USMP. 2014.

2. Reglamento de Evaluación de Pregrado de la FMH -USMP. 2014

3. Hinojoza M. La Tutoría y asesoría entre pares: un binomio en la solución en las universidades. DIDAC 2013; Vol.61 : 45-51

4. Lizaraso F. La Tutoría y Asesoría en la Formación Médica. Horizonte Médico 2013; 13(4):4-5

5. García R. y colaboradores. Impacto del Programa de Tutoría en el desempeño académico de los alumnos del Instituto Tecnológico de Sonora. REDIE 2012 ; 14(1)

6. Fernández Martín, Francisco D. et al. "Prevenión del fracaso académico universitario mediante tutoría entre iguales". Revista Latinoamericana de Psicología 2011; 43 (1): 59-71.

7. García R. y colaboradores. Impacto de la Tutoría presencial y virtual en el desempeño académico de los alumnos universitarios. Revista Iberoamericana de Educación 2012; 58 (2): 1 - 11.
8. Echevarria B. Orientación Profesional. Edit. UOC. 2008

9. Pérez Martín \& Pérez Rodrigo. Las tutorías electrónicas en el horizonte de la formación virtual: un ejemplo desde la filología inglesa. Revista de Educación a Distancia, Universidad de Murcia. España. 2008;19,1-13.

10. Barberá E. Los fundamentos teóricos de la tutoría presencial y en línea: una perspectiva socio-constructivista. 2006.

«tttp:/ / www. carlosruizbolivar. com/ articulos/ archivos/02Los\%2fundamentos\%2te\%C3\%B3ric os\%20de\%20la\%20tutor\%C3\%ADa.pdf>

11. Calvo M. Introducción a la Metodología didáctica. Edit. MAD.S.L. España. 1ra. Edic. 2006.

12. García González, Cecilia, y Olga López Pérez. "Fortalezas de la tutoría entre pares: la experiencia de formarse en la práctica". <http://148.202.105.12/tutoria/encuentro/ files/ ponenciaspdf/ Fortaleza $\% 20 \mathrm{de} \% 201 \mathrm{a} \% 20$ tutoria\%20 entre\%20pares. pdf $>$.

\section{Correspondencia:}

Mauro Rivera Ramírez

Dirección: Av. Alameda del corregidor 1531-

Las Viñas, La Molina. Lima - Perú.

Teléfono: 3652300

Correo electrónico: mriverar@usmp.pe 